\title{
Successful Management of Preterm Premature Rupture of Membrane in Second Trimester: A Case Report and Literature Review
}

\author{
Aparajita Jha, Xiao Li, Shuirong Zhang, Hui Li \\ Department of Obstetrics and Gynaecology, Jingzhou Central Hospital, The Second Clinical Medical College, Yangtze University, \\ Jingzhou, China \\ Email: dr.ajha2011@gmail.com, adsxl-009@126.com,519854672@qq.com,70169683@qq.com
}

How to cite this paper: Jha, A., Li, X., Zhang, S.R. and Li, H. (2019) Successful Management of Preterm Premature Rupture of Membrane in Second Trimester: A Case Report and Literature Review. Yangtze Medicine, 3, 149-156.

https://doi.org/10.4236/ym.2019.32015

Received: March 11, 2019

Accepted: June 25, 2019

Published: June 28, 2019

Copyright $\odot 2019$ by author(s) and Scientific Research Publishing Inc. This work is licensed under the Creative Commons Attribution International License (CC BY 4.0).

http://creativecommons.org/licenses/by/4.0/

\begin{abstract}
The preterm premature rupture of membranes occurring in early pregnancy at less than 23 - 24 weeks (prior to fetal viability), has higher risk for early preterm delivery, and therefore, the poorer the prognosis with poor chance of neonatal survival and a high rate of severe, long-term neonatal morbidity among survivors. In such cases in absence of overt evidence of intrauterine infection at the time of diagnosis termination of pregnancy or expectant management is generally offered modality of treatment, the prior being commonly preffered. When expectant management is instituted, it is very rare that spontaneous resealing of the membranes occurs with the outcome that is equivocal to normal pregnancy. The presented case is an example of this rare happening. A 25-year-old, mangolian, primigravida at 20 weeks of pregnancy had spontaneous preterm premature rupture of membranes. After 8 days of expectant management, she had cessation of amniotic fluid leak and could continue pregnancy till term with normal feto-maternal outcome at 37 weeks of pregnancy. The risk of infection increases with prolongation of latency period but in this case, the latency period was prolonged for more than 16 weeks and there was no evidence of infection, with normal feto-maternal outcome at term. This is the first case of its kind happened in our hospital and deserves to be reported. It is expected that this article will reveal the possibility of resealing of spontaneous preterm premature rupture of membrane with proper expectant management.
\end{abstract}

\section{Keywords}

Preterm, Rupture of Membrane, Expectant Management,

Reseal of Membrane 


\section{Introduction}

Preterm premature rupture of membranes (PPROM) is a common clinical condition with significant impacts on obstetric outcome and is considered one of the "great obstetrical syndromes" responsible for spontaneous preterm birth [1]. The amniotic cavity is a sac made of amnion and chorion known as chorioamniotic membrane. The product of conception in the amniotic cavity is kept safe and sealed by this membrane. Any breech in the integrity of the membrane is rupture of the membrane and the subsequent closer of the breech is considered resealing of the membrane [2]. There have been several techniques developed in an attempt to artificially reseal the fetal membranes and prevent leakage of amniotic fluid, including among others: intra-amniotic injection of platelets and cryoprecipitate (amniopatch), sealing the cervical canal, and fetoscopic laser coagulation. However, there is as yet no effective and safe technique readily available to achieve this goal [3]. It is very rare that resealing occurs spontaneously with rewarding outcome.

PPROM is defined as rupture of the fetal membranes prior to 37 weeks gestation and prior to the onset of labor [4] [5]. The main consequence of PPROM is the onset of premature labor and delivery. Indeed, PPROM accounts for $40 \%$ of all spontaneous preterm deliveries, representing a significant contributor to perinatal morbidity and mortality worldwide [2] [6] [7]. Previable PPROM is defined as spontaneous rupture of membrane occurring between 13 and 23. 6/7 weeks' gestational age is rare, occurring in $1.37 \%$ of twin and $0.52 \%$ of singleton pregnancies [8]. When PROM occurs near the limit of viability, 30 to 40 percent remain pregnant for at least 1 week, and one in five conservatively managed women could continue pregnancy for 4 weeks or more. Only a small proportion of women with membrane rupture can expect cessation of fluid leakage (2.8 to 13 percent), particularly that occuring subsequent to amniocenteis. Approximately 86 percent of those with leakage following amniocentesis will reseal [9]. The resealing of spontaneous PPROM is rare. The case presented here is an example of this rare condition.

\section{Case Report}

A 25-year-old mangolian primigravida at 20 weeks of pregnancy presented to the hospital with chief complaints of watery fluid wetting her undergarment regularly since previous night. It had started spontaneously when she was lying on her bed. She had denied history of pain abdomen, urinary tract infection, vaginal infection, or recent systemic illness, abdominal or pelvic trauma, smoking or other form of tobacco use. There was no history of cervical cerclage or amniocentesis. There was no history of autoimmune or connective tissue disorders like Systemic Lupus Erythematosus or EhlerDanlos Syndrome She was not in labour and there was no (low maternal BMI or) any obvious known maternal risk factor for PPROM and fetal risk factors (like multifetal pregnancy) was also not identified. 
On per abdomen examination-size of uterus was corresponding to the period of amenorrhoea and normal fetal heart sound was audible. Abdomen was non tender. On speculum examination pooling of amniotic fluid from the cervical os was visualized and $\mathrm{pH}$ paper test was used to confirm the diagnosis of premature rupture of membrane.

She was having regular menstrual period with 3 - 5 days bleeding; with 28 days cycle, had her last menstrual period five months back. Pregnancy was confirmed after 9 days of her missed period. The first trimester ultrasound done at 6 weeks of pregnancy showed gestational age appropriate for the period of amenorrhoea. She was perceiving normal fetal movement and denied history of pain abdomen or bleeding per vagina. Her past medical and surgical history was not relevant. She was afebrile with no abnormality detected on systemic examination She was admitted to the hospital. She was explained about the risks and benefits of active and expectant management. She insisted on the expectant management. She was managed expectantly with antibiotic and modified bed rest, advising her to keep the activity limited to basic self care. She was given injection Ampicillin $2 \mathrm{gm}$ and injection Erythromycin $500 \mathrm{mg}$, intravenously, 6 hourly for 2 days followed by Amoxycillin $500 \mathrm{mg}$ and Erythromycin $250 \mathrm{mg} 8$ hourly, orally for 8 days. She was advised to lie on bed with elevation of hip and lower part of the body about six centimeters above the level of upper part of the body, to minimize leaking-modified bed rest, as in pelvic rest she was advised to avoid use of tampons, douching, or intercourse. During her hospital stay routine blood (complete blood count) and urine examination was done and the reports were normal; she was evaluated for presence of infection by CRP test every alternate day-it was negative every time.

On laboratory investigation: Blood group was "A" Rhesus-Positive, Hb was $11.6 \mathrm{gm} / \mathrm{dL}$ (Normal range: $12-16 \mathrm{~g} / \mathrm{dL}$ ), Hematocrit was 35\% (Normal range: $36 \%$ - 47\%), RBC was $4.1 \mathrm{million} / \mathrm{mm}^{3}$ (Normal range: 4.5 - $5.5 \mathrm{million} / \mathrm{mm}^{3}$ ), WBC was $6000 / \mu \mathrm{L}$ (Normal range: 4000 - 10,000/ $\mu \mathrm{L}$ ), Platelet count was $230,000 / \mu \mathrm{L}$ (Normal range:150,000-350,000/ $\mathrm{L}$ ), Prothrombin time was $10.50 \mathrm{~s}$ (Normal range: $10-13 \mathrm{~s}$ ), INR was $1 \mathrm{~s}$ (Normal range: $0.80-1.20 \mathrm{~s}$ ), Fibrinogen was $4.10 \mathrm{~g} / \mathrm{L}$ (Normal range: $2.38-4.98 \mathrm{~g} / \mathrm{L}$ ), serum creatinine was 0.7 (Normal range: $0.7-1.3 \mathrm{mg} / \mathrm{dL}$ ), Random plasma glucose was $87 \mathrm{mg} / \mathrm{dl}$ (Normal range: $70-140 \mathrm{mg} / \mathrm{dL}$ ), CRP (C-Reactive Protein) was Negative every time, Hepatitis B surface Antigen was Negative, Hepatitis B surface Antibody was Negative, Hepatitis B e Antigen was Negative, HB e Antibody was Negative, HB c Antibody was Negative, HCV Antibody was Negative, HIV was Negative, VDRL was Non-Reactive. On urine examination: Color was yellow, Transparency was clear, Bacterial sugar was Negative, Ketone bodies was Negative, Urine specific gravity was 1.018 (Normal range: $1.003-1.030$ ), Acidity/Alkalinity (pH) was 6.5 (Normal range: 4.5 - 8.0), Urine protein was Negative, Nitrite was Negative, Occult blood was Negative, Leucocyte esterase was Negative, Urine culture-No growth was seen after 24 hours of incubation, High vaginal swab culture-No growth was seen after 24 hours of incubation. The ultrasound finding of AFI $9.4 \mathrm{~cm}$ on first 
day and $3.2 \mathrm{~cm}$ on fourth day is supportive evidence for the diagnosis of PPROM. Figure 1 (The dotted line represents amniotic fluid column). This is the initial ultrasound image showing adequate amount of amniotic fluid. Figure 2 (The dotted line represents amniotic fluid column). This is the ultrasound image after three days of leaking - showing decreased amount of amniotic fluid. The comparision of above two images shows that there was significant amount of amniotic fluid loss. During observation she was regularly examined for signs of intrauterine infection. The amount of amniotic fluid leak was estimated by visual assessment of wet bed sheet, wet clothing and wet pad; also evaluated for color and smell to rule out anything other than amniotic fluid; it was found to be gradually decreasing after initial three days.



Figure 1. (The dotted line represents amniotic fluid column.) This is the initial ultrasound image showing adequate amount of amniotic fluid.

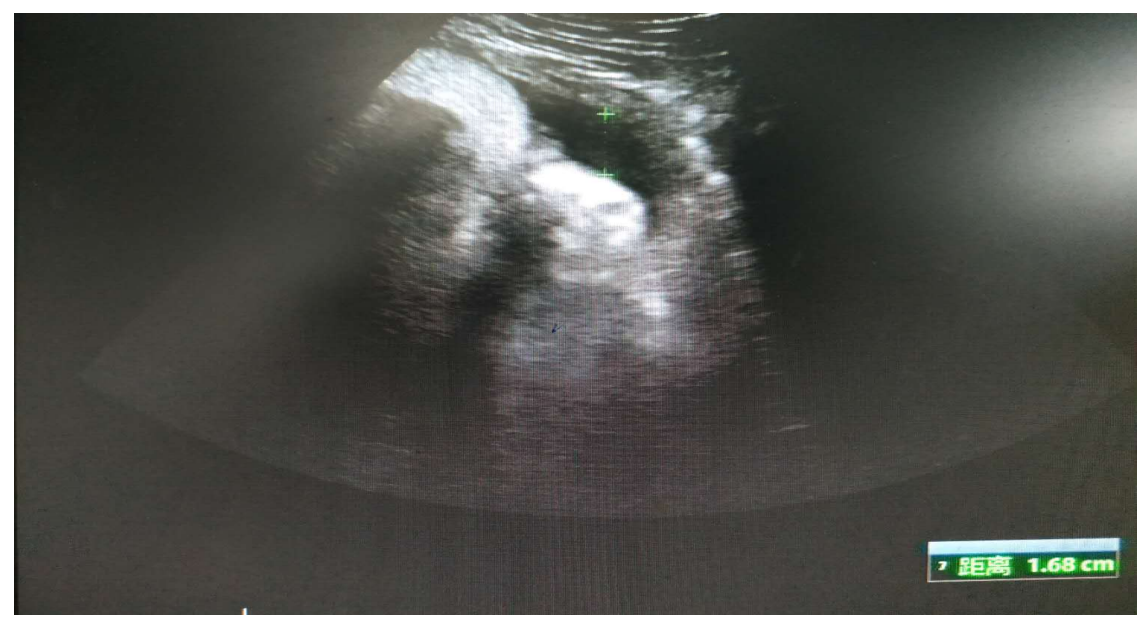

Figure 2. (The dotted line represents amniotic fluid column.) This is the ultrasound image after three days of leaking - showing decreased amount of amniotic fluid. The comparision of above two images shows that there was significant amount of amniotic fluid loss. 
The ultrasound finding of AFI $9.4 \mathrm{~cm}$ on first day and $3.2 \mathrm{~cm}$ on fourth day is supportive evidence for the diagnosis of PPROM. On 8 day of expectant management, there was cessation of amniotic fluid leak. She was kept under observation for another 2 days when she was off the bed rest, moving normally for day-to-day activities. Then she was absolutely free of leaking and was discharged after 10 days of her hospital stay. On discharge she was advised to maintain pelvic rest and keep herself at ease. This patient being aware of the risk of preterm delivery, she was following the advice properly, as she said in her subsequent antenatal visit. There after she had uneventful pregnancy till term.

At 37 weeks of pregnancy cesarean section was done, on patient's request, though there was no obstetric indication. The outcome was male fetus of $3.2 \mathrm{~kg}$ birth weight with apgar score of $8 / 10$ and $9 / 10$ at 1 minute and 5minute respectively. The babys routine blood test and CRP test was done; infection ruled out, sucking and all the reflexes were normal; baby declared normal by paediatric doctor. The mother had normal recovery.

\section{Discussion}

Currently, there is no statistical evidence to support particular therapeutic approach for previable or early preterm premature rupture of membranes (EPPROM) occurring between 14 and 24 WG (weeks gestation) before viability of the fetus. According to some literature the common approach is expectant management hoping to reach a viable period from $23-24$ WG in order to use corticosteroids, antibiotics and transfer to level III ward. [10] For the same case some literature suggests termination of pregnancy to be justifiable since the survival of fetus in case of PROM $<22$ weeks is significantly lower (14.4\%) than $\geq 22$ weeks (57.7\%). According to ACOG, survival rates are apparently overestimated. The risk needs to be individualized and depends on the combination of birth weight, gestational age and sex of the fetus which will impact morbidity/mortality.

Termination of pregnancy is considered because this condition of PROM $<22$ weeks is known to be associated with pulmonary hypoplasia which is incompatible with life for fetal survival. The approximate incidence of pulmonary hypoplasia being $10 \%-20 \%$ in such cases. There is insufficient data to rely upon ultrasound for determination of lung volumes or function. The risk of complications associated with prematurity that commonly includes respiratory distress syndrome, intraventricular haemorrhage, necrotizing enterocolitis, is constantly present [11].

Oneretrospective study was conducted (by J. M. Bendix et al.) in a tertiary referral unit at Rigshospitalet, University of Copenhagen, to identify risk factors of major complications following PPROM and if those complications were predictable; out of 234 women with PPROM between 22 and 33 weeks of gestation, the complication rate in early PPROM before 28 weeks of gestation was $64 \%$, much higher compared to $11 \%$ in late PPROM at 28 weeks of gestation or later. 
This study indicates that the risks of complications are expected to be still higher in case of PPROM in less than 22 weeks of gestation [12].

As mentioned in a case report of 31 year-old second gravida, para 1 had PPROM on next day of genetic amniocentesis at 17 weeks of gestation, the diagnosis was confirmed using standard technique and she was admitted for expectant management. In that case, reseal occurred in 48 hours after evident PROM. AFI was found to be increased to $5.4 \mathrm{~cm}$ from $1.8 \mathrm{~cm}$. Then pregnancy was uneventful till 33 weeks of gestation when placenta previa totalis was diagnosed and cesarean section was done with normal fetal and maternal outcome [13].

In most patients with preterm PROM following amniocentesis, the membranes reseal with restoration of normal amniotic fluid volume. This situation is different from spontaneous preterm PROM in which apparent resealing of the membranes and reaccumulation of normal amniotic fluid volume is far less common (estimated at $2.8 \%-13 \%$ ), especially if it has not occurred within 72 hours of presentation. This incident provokes us to think about reconsideration of the mode of active management from immediate delivery to artificial resealing of membrane in such condition [3].

It is also possible that interventions that impact latency in PPROM may be gestational age dependent [8].

Even with the standard expectant management $40 \%$ - 50\% will deliver within 1 week and $70 \%-80 \%$ will deliver within 2 - 5 weeks. This may not improve the outcome significantly but is associated with maternal complications like infection, endometritis, abruption, retained placenta and maternal sepsis risk of $1 \%[11]$.

As mentioned in an Intervention Review regarding sealing procedures for PPROM (by Adele E Crowley1, et al.) based on two small trials. From one trial (including 35 women), no clear difference was observed between the mechanical sealing group and the control group in relation to neonatal sepsis or chorioamnionitis. Though it was small trial (involving 94 women) oral immunological membrane sealant was found to be associated with decreased incidence of preterm birth and neonatal death [14].

In case of PPROM when expectant management is strongly desired by the patient even after understanding the consequences the role of artificial techniques to reseal the membrane (like intra-amniotic injection of platelets and cryoprecipitate/amniopatch, sealing the cervical canal, and fetoscopic laser coagulation) appears to be vital but so far such techniques are not readily available in clinical practice. So, the mainstay of expectant management remains to be administration of antibiotics and modified bed rest [3].

The patient in the presented case was counseled regarding risks and benefits of expectant management vs. immediate delivery. Immediate delivery was offered as an option to overcome the likely complications. But the patient insisted on expectant management and she was managed accordingly. To our surprise there was no amniotic fluid leak after 8 days of expectant management 
and reaccumulation of amniotic fluid in the subsequent ultrasound was indicating the reseal of the membrane. The latency period of more than 16 weeks was achieved with normal feto-maternal outcome at term.

As mentioned in one review of current evidence of fetal membrane healing, amnion-derived cell monolayers were found to have repair capacity which varied with the period of gestation. In vitro it was proved that those monolayers obtained at earlier gestational ages showed higher proliferation rates with faster closure of the central defect [15].

Another scientific report on Healing of PROM (by Haruta Mogamil, et al.) also mention that small ruptures of the fetal membrane closed within $72 \mathrm{~h}$ whereas healing of large ruptures was only $40 \%$, as observed in preclinical mouse model [16].

In absence of active healing of the ruptured membranes, reseal could occur through retraction, sliding, contraction, and scarring in the myometrial and decidual layers of the uterus [15].

\section{Conclusion}

Such a case suggests that preterm premature rupture of membrane can reseal spontaneously with proper expectant management. The literature suggests that most of the anatomical and physiological changes during pregnancy are directed towards maintaining pregnancy for good fetal outcome. The resealing of PPROM following amniocentesis in $86 \%$ of the cases is the evidence of this physiological mechanism of healing. The mechanism involved in spontaneous resealing of ruptured membrane and the factors that promote it remains yet to be investigated.

\section{Conflicts of Interest}

The authors declare no conflicts of interest regarding the publication of this paper.

\section{References}

[1] Lee, J.H., Romero, R., Kim, S.M., Chaemsaithong, P., Park, C.-W., Park, J.S., Jun, J.K. and Yoon, B.H. (2016) A New Antimicrobial Combination Prolongs the Latency Period, Reduces Acute Histologic Chorioamnionitis and Funisitis, and Improves Neonatal Outcomes in Preterm PROM. The Journal of Maternal-Fetal \& Neonatal Medicine, 29, 707-720. https://doi.org/10.3109/14767058.2015.1020293

[2] Fortner, K.B., Grotegut, C.A., Ransom, C.E., Bentley, R.C., Feng, L., et al. (2014) Bacteria Localization and Chorion Thinning among Preterm Premature Rupture of Membranes. PLOS ONE, 9, e83338. https://doi.org/10.1371/journal.pone.0083338

[3] Caughey, A.B., Robinson, J.N. and Norwitz, E.R. (2008) Contemporary Diagnosis and Management of Preterm Premature Rupture of Membranes. Reviews in Obstetrics \& Gynecology, 1, 11-22.

[4] Wong, L.F., Holmgren, C.M., Silver, R.M., Varner, M.W. and Manuck, T.A. (2015) Outcomes of Expectantly Managed Pregnancies with Multiple Gestations and Preterm Premature Rupture of Membranes Prior to 26 Weeks. American Journal of 
Obstetrics \& Gynecology, 212, 215.e1-215.e9. https://doi.org/10.1016/j.ajog.2014.09.005

[5] Amelie, K., Cardoso, G.P., Thill, C., Verspyck, E. and Marre, S. (2016) Outcome at Two Years of Very Preterm Infants Born after Rupture of Membranes before Viability. PLoS ONE, 11, e0166130. https://doi.org/10.1371/journal.pone.0166130

[6] Yee, L.M. and Grobman, W.A. (2016) Perinatal Outcomes in Cephalic Compared with Noncephalic Singleton Presentation in the Setting of Preterm Premature Rupture of Membranes Prior to 32 Weeks of Gestation. Obstetrics \& Gynecology, 128, 812-818. https://doi.org/10.1097/AOG.0000000000001614

[7] Romero, R., Miranda, J., Chaemsaithong, P., Chaiworapongsa, T., Kusanovic, J.P., Dong, Z., Ahmed, I.A., Shaman, M., Lannaman, K., Yoon, B.H., Hassan, S.S., Kim, C.J., Korzeniewski, S.J., Yeo, L. and Kim, Y.M. (2015) Sterile and Microbial-Associated Intra-Amniotic Inflammation in Preterm Prelabor Rupture of Membranes. The Journal of Maternal-Fetal \& Neonatal Medicine, 28, 1394-1409. https://doi.org/10.3109/14767058.2014.958463

[8] Myrick, O., Dotters-Katz, S., Grace, M., Manuck, T., Boggess, K. and Goodnight, W. (2016) Prophylactic Antibiotics in Twin Pregnancies Complicated by Previable Preterm Premature Rupture of Membranes. American Journal of Perinatology Reports, 6, e277-e282. https://doi.org/10.1055/s-0036-1587324

[9] Gabbe, S.G., Niebyl, J.R. and Simpson, J.L. (2007) Premature Rupture of the Membranes. In: Gabbe, S.G., Ed., Obstetrics: Normal and Problem Pregnancies, Fifth Edition, Mosby Elsevier, London, Chapter 27, 713-732.

[10] Brookfield, K.F., El-Sayed, Y.Y., Chao, L., Berger, V., Naqvi, M. and Butwick, A.J. (2015) Antenatal Corticosteroids for Preterm Premature Rupture of Membranes: Single or Repeat Course? American Journal of Perinatology, 32, 537-544. https://doi.org/10.1055/s-0034-1396690

[11] Gross, S.J. ACOG Guidance Update Diagnosis and Management of PROM (Prelabor Rupture of Membranes).

https://www.obgproject.com/2017/12/29/acog-guidance-update-diagnosis-manage ment-prom-prelabor-rupture-membranes/

[12] Bendix, J.M., Hegaard, H.K., Bergholt, T. and Langhoff-Roos, J. (2015) Expectant Management of PPROM and Major Complications before Planned Delivery: A Retrospective Cohort Study. Journal of Obstetrics and Gynaecology, 35, 570-577. https://doi.org/10.3109/01443615.2014.987114

[13] Phupong, V. and Ultchaswadi, P. (2006) Spontaneous Reseal of Ruptured Membranes after Genetic Amniocentesis. Journal of the Medical Association of Thailand, 89, 1033-1035.

[14] Crowley, A.E., Grivell, R.M. and Dodd, J.M. (2016) Sealing Procedures for Preterm Prelabour Rupture of Membranes. Cochrane Database of Systematic Reviews, 7, CD010218. https://doi.org/10.1002/14651858.CD010218.pub2

[15] Lin, G.G. and Scott, J.G. (2012) Fetal Membrane Healing after Spontaneous and Iatrogenic Membrane Rupture: A Review of Current Evidence. NIH Public Access, 100, 130-134.

[16] Mogami, H., Hari Kishore, A., Akgul, Y. and Word, R.A. (2017) Healing of Preterm Ruptured Fetal Membranes. Scientific Reports, 7, Article No. 13139. https://doi.org/10.1038/s41598-017-13296-1 